\title{
Visuomotor transformations for eye-hand coordination
}

\author{
D.Y.P. Henriques, W.P. Medendorp, A.Z. Khan and J.D. Crawford* \\ York University, Centre for Visual Research, Departments of Psychology and Biology, 4700 Keele St., BSB, rm 291, \\ Toronto, ON M3J 1P3, Canada
}

\begin{abstract}
In recent years the scientific community has come to appreciate that the early cortical representations for visually guided arm movements are probably coded in a visual frame, i.e. relative to retinal landmarks. While this scheme accounts for many behavioral and neurophysiological observations, it also poses certain problems for manual control. For example, how are these oculocentric representations updated across eye movements, and how are they then transformed into useful commands for accurate movements of the arm relative to the body? Also, since we have two eyes, which is used as the reference point in eye-hand alignment tasks like pointing? We show that patterns of errors in human pointing suggest that early oculocentric representations for arm movement are remapped relative to the gaze direction during each saccade. To then transform these oculocentric representations into useful commands for accurate movements of the arm relative to the body, the brain correctly incorporates the three-dimensional, rotary geometry of the eyes when interpreting retinal images. We also explore the possibility that the eye-hand coordination systems uses a strategy like ocular dominance, but switches alignment between the left and right eye in order to maximize eye-hand coordination in the best field of view. Finally, we describe the influence of eye position on eye-hand alignment, and then consider how head orientation influences the linkage between oculocentric visual frames and bodycentric motor frames. These findings are framed in terms of our 'conversion-on-demand' model, which suggests a virtual representation of egocentric space, i.e. one in which only those representations selected for action are put through the complex visuomotor transformations required for interaction with actual objects in personal space.
\end{abstract}

Keywords: Eye-hand coordination; Visuospatial updating; Eye dominance; Retinal geometry; Eye position; Visuomotor transformation; Head position; Body geometry

\section{Introduction}

Eye-hand (or hand-eye) coordination is a topic that just about everyone can relate to. We need good eyehand coordination to reach out and pick up a coffee cup, press a doorbell, or catch a ball. Indeed many of the skilled activities that distinguish humans from other species involve good eye-hand coordination.

\footnotetext{
* Correspondence to: J.D. Crawford, York University, Centre for Visual Research, Departments of Psychology and Biology, 4700 Keele St., BSB, rm 291, Toronto, ON M3J 1P3, Canada. Tel.: +1-416-736-2100 ext. 88641; Fax: +1-416-736-5814; E-mail: jdc@yorku.ca
}

Most of these we take for granted - only in cases of extreme athletic prowess do we notice eye-hand coordination. But these more basic abilities become all too significant when they are impaired, as in the neurological patients studied by Carey et al. (2002, this volume).

Given its centrality in our lives, it is not surprising that the study of this behavior has a long history (as early as Rene Descartes). Clearly this has remained a major topic of study, with various scientific contributions made in major laboratories of the 20th century. And yet at this time one will find no erudite 'Society for Eye-Hand Coordination', or 'Annual Meeting for Eye-hand Coordination'. However, as the contributions to this section of the current volume testify, 
it would seem that it is a topic which has reached its day, perhaps because we know just enough about these various sub-systems to now try putting them altogether.

Our particular interest is in the geometric aspects of the forward serial transformations within the brain that use vision to guide movement of the hand. Although many studies of eye-hand coordination look at the input (vision) and the output (hand movement), our focus is on the transformations that account for all of the linkages in between - from eye, to head, to body, to arm, to hand. Most of our studies are on pointing or 'touching', a pared-down version of eye-hand coordination minus the grasp element. Our goal is to build up a rigorous model - the kind that could actually control such a system if one were to build it from scratch. Our belief is that in building up such a model, we will gain a clearer understanding of the neural processes that one should look for in the brain. The following is not a general review of the topic: in the short space allowed here our aim is mainly to summarize some of our own work in this area, and to show how this work has motivated our thinking in this area.

\section{Eyes on the prize}

Many studies, including those described in the other contributions to this section and elsewhere (e.g. Gielen et al., 1984; Fisk and Goodale, 1985; Vercher et al., 1994; Engel et al., 2000) have demonstrated the intimate coupling between movements of the eyes and hand. But why do we need this? What is the advantage of coupling the eye and hand? One way to find out is to de-couple the system and see what happens, i.e. ask people to reach or point toward objects that they are not looking at. Under these conditions, Roland Johansson and colleagues have shown how reaching/manipulating movements that are normally precise become fumbling approximations. We wanted to quantify the effects of uncoupling on pointing performance (Henriques et al., 1998; Henriques and Crawford, 2000). This work followed from earlier observations by Bock (1986) and Enright (1995), showing that people point past remembered targets located in their visual periphery, which we confirmed and expanded on.

We found that when forced to point toward a central target in the absence of visual feedback, with the eyes deviated, subjects showed systematic misjudgments of target direction. Individual subjects showed different patterns of error, which varied rather anisotropically depending on the direction of the target relative to gaze. But overall, subjects showed a consistent pattern: a tendency to overestimate the distance of the target relative to gaze (Fig. 1A). When asked to direct their gaze $30^{\circ}, 15^{\circ}$, and $5^{\circ}$ both to the right and left of the pointing target, subjects' past-pointing increased with the amount of gaze deviation until saturating at about $2-4^{\circ}$ error at $15^{\circ}$ gaze deviation. Further controls - in which both the gaze target and pointing target were varied - confirmed Bock's finding that this effect was related to the amount of retinal deviation of the target, not eye position per se (Bock, 1986).

Why this degradation in performance? One obvious reason is that foveal vision has a much higher acuity. However this cannot be the full answer, because retinal acuity $5-15^{\circ}$ from the fovea is still too good to explain the poor performance in our studies. Another possibility is that a 'gaze-guidance' system can use the line of sight as a special cue to guide the hand toward foveated targets. This might somehow account for the magnetic reaching behavior seen in Carey's patient (see Carey et al., 2002, this volume). But in another study where our subjects placed their finger onto a remembered target site (Henriques et al., 2001) they did not always look correctly onto the target, but these errors in fixation did not correlate with errors in reaching. For instance, when subjects looked slightly to the left of the target, they were no more likely to misreach to the left than to the right. Thus, contrary to what might be predicted by a gazeguidance hypothesis, small, unconscious deviations in gaze do not drag the hand with them, though they do cause small non-specific degradations in performance. Instead, subjects may misreach more when their gaze misses the target site because the target falls outside the foveal part of vision. Thus, our favored explanation is that the system is simply better calibrated for foveated targets, and that conversely the neural outputs of other patches of retina are poorly calibrated for eye-hand coordination. This does not explain the specific overshooting pattern that we found, but that is a topic we will take up again in a later section. 


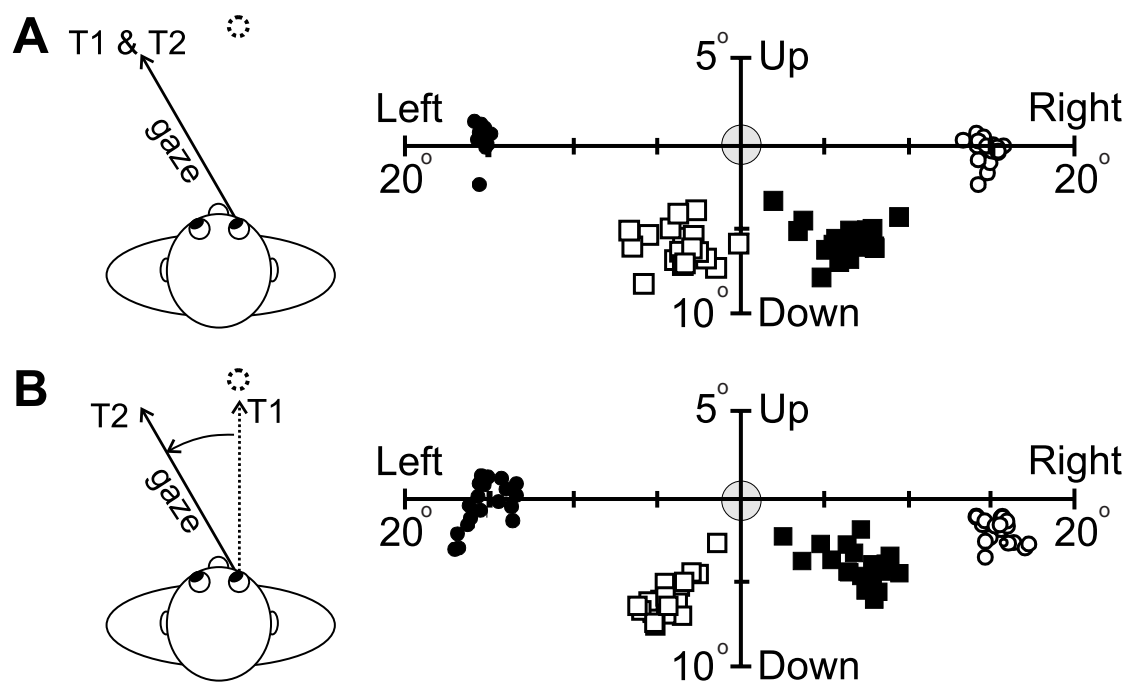

Fig. 1. Final 2-D pointing directions (squares) and gaze direction (circles) relative to central target for a head-fixed subject. Left column: the two tasks, where subjects either view the target peripherally (A) or foveates the target (T1) before looking also to the left (T2) (B). Right column: in the top task (A), the subject past-points in the direction opposite to gaze; this is due to an overestimation of the retinal eccentricity of the target. In the bottom task (B), the subject also past-points in the direction opposite to their final gaze direction, although they fixated the target first (this paradigm is further illustrated in Fig. 2, middle column). Open symbols indicate $15^{\circ}$ rightward fixation trials; solid symbols indicate $15^{\circ}$ leftward fixation trials. Modified from Henriques et al., 1998.

\section{Now you see it, now you don't}

A visuomotor puzzle is how it is that we can look at a target, look away from it so that it is out of sight, and still know where the target is (Hallett and Lightstone, 1976; Mays and Sparks, 1980). Clearly, a fixed retinal impression of the target location would be insufficient and downright misleading, so the brain must be doing something more sophisticated.

One idea was that the brain builds up representations of space by comparing vision with eye position, head position, and so on (Flanders et al., 1992). Unfortunately, the neurophysiological evidence for this mechanism, at least in visuomotor transformations, remains somewhat sketchy, boiling down to some fairly subtle eye position signals (Andersen and Mountcastle, 1983; Schlag et al., 1992; Graziano et al., 1994; Bremmer et al., 1999; Jouffrais and Boussaoud, 1999) with no clear maps of head-centered or body-centered space. Perhaps these maps are distributed in some way (Bremmer et al., 1999). But a more recent suggestion, consistent with certain signals recorded in the visuomotor structures of the brain (Duhamel et al., 1992; Mazzoni et al., 1996), suggests that each time the eyes move, an internal copy of this movement is used to remap our internal on-line representations of visual space in a retinal frame.

The gaze-dependent pointing errors described in the previous section (Fig. 1A) provided the opportunity to test between these mechanisms in the eyehand coordination system (Fig. 2). We reasoned that since subjects make pointing errors as a function of retinal eccentricity (as described above), then they should make these same errors when redirecting their gaze produces a similar 'retinal eccentricity' for the remembered fixated target site - as if their internal representations were remapped to the same retinal location during an eye movement (Fig. 2, right column), even if they looked at the target with the fovea (Henriques et al., 1998). For instance, if subjects point past a remembered target seen while they are looking $15^{\circ}$ to its left, they should also past-point after they move their eyes to $15^{\circ}$ left subsequent to fixating the flashed target. On the other hand, if subjects formed static, head- or body-centered representations of targets (Fig. 2, left column) then pointing based on an initially foveated target should 


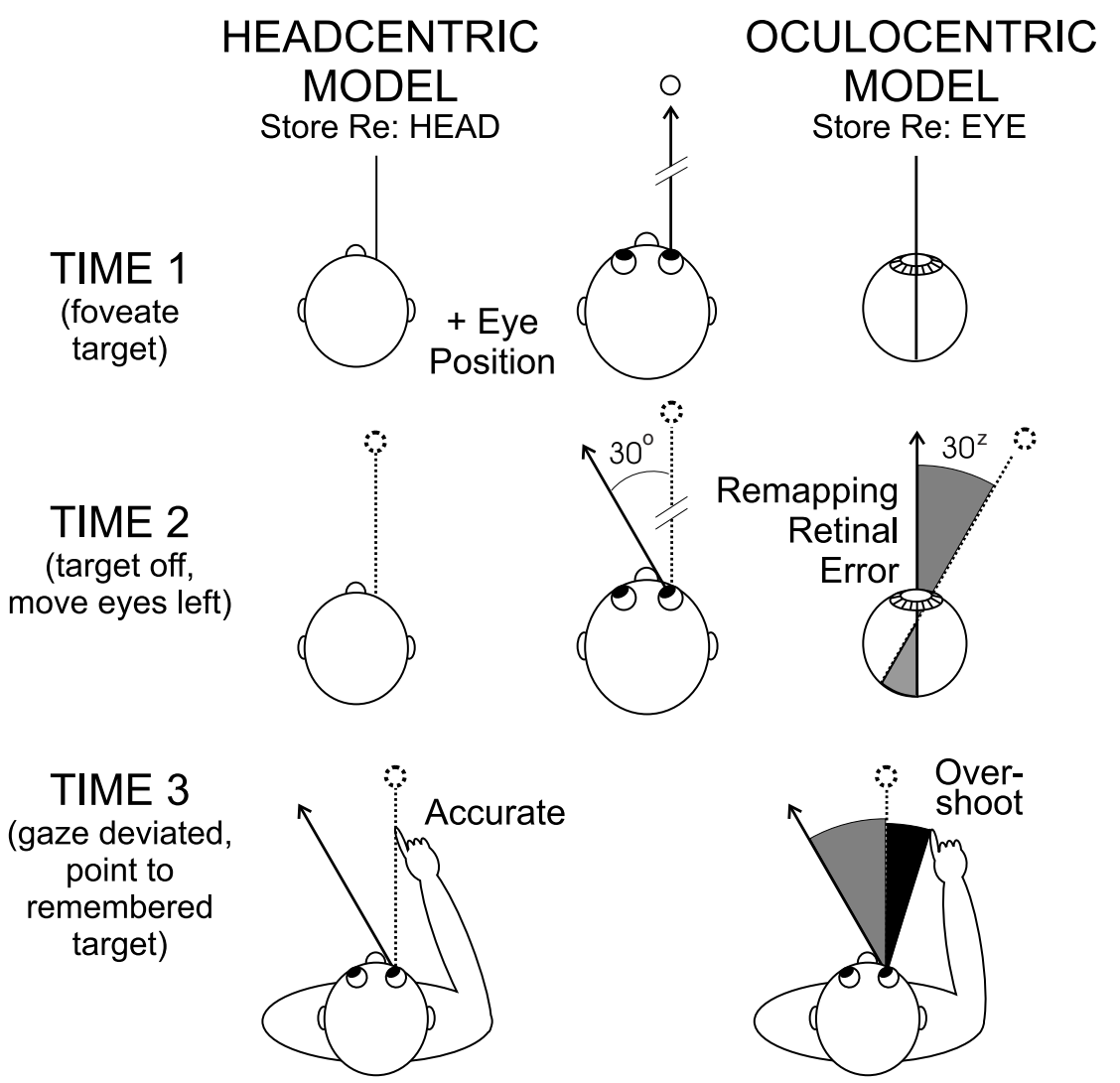

Fig. 2. Predictions of the headcentric (left) and oculocentric (right) models of visuospatial memory on subjects' pointing to a remembered target. The test paradigm (shown in the middle, as well as in Fig. 1B), has subjects look at the target (time 1) and then look $30^{\circ}$ left after the target disappears (time 2) before pointing to its remembered location (time 3). The key feature of this test is that during the visuomotor transformation for pointing, subjects usually exaggerate the retinal eccentricity of the remembered direction of non-foveal targets (top row of Fig. 1, also Bock, 1986; Enright, 1995). The headcentric model holds that we compute target direction relative to the head (by combining retinal signals with eye position) as soon as we fixate the target (in time 1). Note that retinal eccentricity at time 1 is zero and therefore is not subject to the exaggeration effect. According to the headcentric model, this head-centered memory trace remains stable during the intervening eye movement at time 2 , so that accurate pointing is predicted at time 3 . The oculocentric model holds that the target is coded relative to current gaze direction and as a result the leftward eye movement at time 2 must be compensated for, by the counter-rotating of the retinotopic memory trace $30^{\circ}$ to the right (time 2 , right panel). Now the subject must point based on a peripherally shifted retinotopic memory trace, which is susceptible to the exaggeration effect. Therefore, the oculocentric model predicts subject will past-point in the direction opposite to the final gaze direction. Modified from Henriques et al., 1998.

not be affected by subsequent eye movements (that, indeed is the point of this model).

In summary, in the paradigm illustrated both in Fig. 1BFig. 2, where people redirect their gaze away from the fixated target before pointing to its remembered location, a head-centered model would predict accurate open-loop pointing (Fig. 2, bottom left), whereas an eye-centered remapping model would predict that subjects past-point in the direction opposite to gaze (Fig. 2, bottom right) like they do when they point to the remembered location of a peripherally viewed target (as shown by the data in Fig. 1A). Note that neither model explains pastpointing in the peripherally viewed target condition shown in Fig. 1A, but given this phenomena, the two models predict different pointing performance for the paradigm shown in Fig. 2. Thus, the results in Fig. 1A merely provides the necessary control for pointing based on peripheral retinotopic representations. 
Our results clearly favored the eye-centered remapping model (Fig. 1B). When subjects foveated a briefly flashed target in complete darkness, and then deviated their eyes, they did not point accurately as they did when they maintained gaze on the remembered target site throughout the trial. Instead, they made the same errors in pointing as if they had viewed the target in the new retinally peripheral location (compare Fig. 1B to Fig. 1A). In other words, it looks like they were pointing based on a shifted, retinotopic representation. Based on this result, we concluded that the eye-hand coordination system uses this same mechanism (Henriques et al., 1998), which had previously been proposed and described for the oculomotor system. Shortly afterwards Andersen and colleagues (Batista et al., 1999) discovered that single-unit responses are consistent with such a mechanism in the parietal reach region (PRR) - an arm control center with retinotopically organized receptive fields.

\section{Near vs. far}

The previously described study by Henriques et al. (1998) was done exclusively with pointing targets that were well beyond reach, in so-called extrapersonal space. However, a number of neuropsychological studies have suggested that different neural mechanisms are used for coding near (peripersonal) space (for a review see Colby and Goldberg, 1999). This makes some sense for eye-hand coordination. Anything within reach is coded by preparatory activity in primary motor cortex (M1), whose signals are clearly not organized in eye-centered coordinates (Fu et al., 1993; Riehle and Requin, 1995; Mushiake et al., 1997; Crammond and Kalaska, 2000). Why not code for near targets using the stable, musclecentered, eye-movement independent codes of M1?

To test which spatial mechanism dominates human reaching behavior, across eye movements, in near space, we repeated the paradigm in Henriques et al. (1998) (Fig. 2), but this time using three sets of arm pointing/reaching targets - one beyond reach, one at $42 \mathrm{~cm}$, and one at $15 \mathrm{~cm}$. According to the hypothesis that near and far space are coded differently, subjects should have shown the same result as before for the far target, but should have shown a more stable reaching response for the near targets, unaffected by the intervening eye movement. But this is not what happened (Medendorp and Crawford, 2002). Instead, subjects showed the same effect for all three-target sets: that predicted by the eyecentered remapping model. It would be a mistake to conclude that this means that the muscle/body centered representations are never used to code near space in M1 and other structures, but this result does suggest structures like PRR which do utilize remapping override those responses, updating them after each eye movement. Perhaps, the near-far distinction may be more relevant for perception than for action. If so, our results support the notion that target locations are remembered by shifting retinotopic representations as a function of each eye movement, and suggest that this is a general spatial mechanism for near and far space.

\section{Cyclops had it easy}

When we speak of eye-hand coordination, we immediately propagate an implicit error: only a mythical one-armed Cyclops has eye-hand coordination. Most of us have to coordinate two eyes with two hands, but one generally chooses one hand in reaching, often the dominant hand. But which eye is chosen? Or is it some synthesis of the two?

This gets us into the sticky territory of ocular dominance and the cyclopean eye. Ocular dominance has many meanings. Here we consider just alignment of the hand with the eye. For example, in our monocular pointing studies, we have found that subjects tend to align the fingertip between the eye and the target, as if they were reaching out to touch it (as opposed to aligning the line of the arm to the target). So, which eye do they choose? In neurophysiological terms, which eye dominates the eye-centered representations in the brain described above?

One appealing idea, put forward in the 19th century by Wells and more recently championed by Ono (Wells, 1792; Ono and Barbeito, 1982), is that the inputs from the two eyes are synthesized and referenced to a virtual 'cyclopean eye' positioned between them. But, even this theory would require the fingertip to be positioned along the line of sight of one eye or the other to be perceived as dead ahead of the cyclopean eye (this may sound contradictory, but makes sense in terms of Wells' original exper- 
iments). Thus, the question arises again, which eye does the hand coordinate with?

A number of classical studies suggest that the hand prefers to align with a dominant eye (Miles, 1930; Crider, 1944; Coren and Kaplan, 1973; Porac and Coren, 1976). However, we noted that all of these studies were done with targets located straight ahead. But what about more peripheral targets? Would it not make sense for the system to be more flexible, and chose either eye, depending on which one had the better field of view?

To test this idea, we used a variation of an old paradigm (Crider, 1944) where we had subjects reach out and grasp a ring while visually fixating a target through that ring, and then bring the ring back to 'the eye' without allowing it to cross the line of sight (Fig. 3). Subjects are expected to choose the 'dominant' line of sight, and indicate this by bringing the ring back to the corresponding eye. Their performance on this task proceeded as we had expected (Khan and Crawford, 2001a): subjects tended to choose the right eye for rightward (but still binocular) targets, and the left eye for leftward targets (Fig. 4). The switch in choice generally occurred surprisingly close to, but just off, center, so that if subjects had been tested only at the center target they would have appeared to be either left eye or right eye dominant. In a similar, more recent test of this hypothesis, we asked subjects to point toward targets at various horizontal eccentricities, and then examined the kinematics of their arm position to determine if the hand had been aligned with the left or right eye (Khan and Crawford, 2001b). Again, we found a clear tendency for subjects to choose the left eye for leftward targets and vice versa, although curiously this tendency was reduced by visual feedback. This may be a motor strategy that allows the hand to coordinate with either one eye or the other in way that optimizes vision, perhaps relying on eye position signals or some other cue.

\section{The importance of knowing eye position}

Speaking of eye position signals, how does the eyehand coordination system transform eye-centered visual signals into useable commands for arm movement? At first glance, the eye-centered remapping mechanism described in the previous sections would
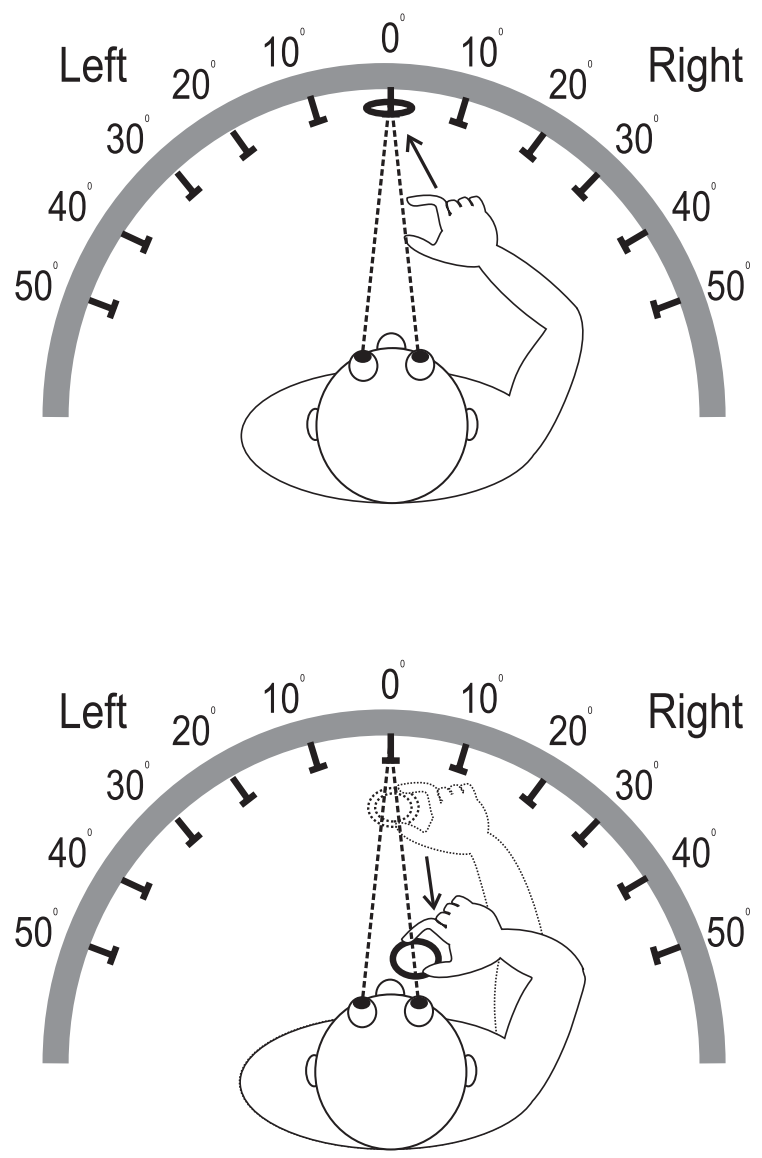

Fig. 3. Illustration of a reach-grasp task to test whether ocular dominance shifts between the eyes as a function of gaze direction. Method: subjects were instructed to fixate on one of 11 rings placed horizontally $\left(50^{\circ}\right.$ left and right of center) in a semi-cylinder, and grasp the ring (top) and bring it all the way back to their face is a smooth fluid motion, without allowing it to cross either line of gaze (bottom). Modified from Khan and Crawford, 2001a.

seem to obviate the need to continuously take eye position into account. But that is only true with regards to the mechanism for coding and storing spatial memory described in those sections. Once one has to actually act on those representations, the eye positions signals become vital.

Why is this so? Supposing that one always started off by looking and pointing in the same direction, one might think it a simple matter to drive the arm along the arc in parallel with the displacement of the target as coded on the retina, or the target's retinal displacement. To code the arm movement, the brain 

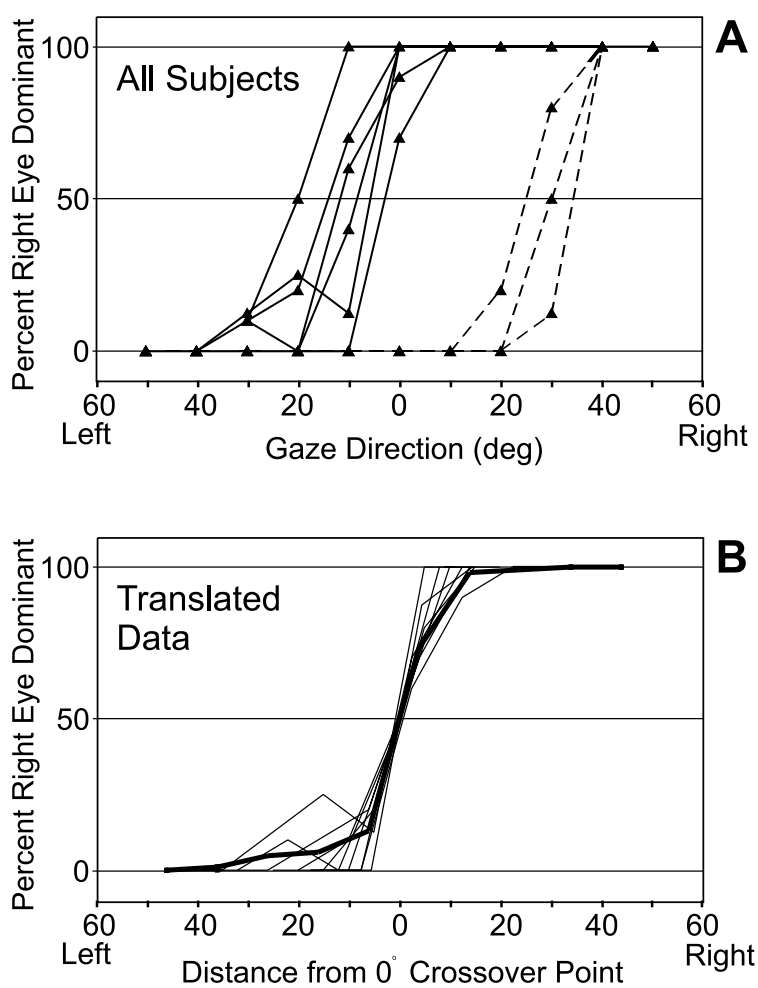

Fig. 4. Gaze position dependence of ocular dominance. (A) The percentage of trials where the right eye was dominant $(0 \%$ indicates that the left eye was always dominant) is shown for each gaze/target direction for 10 subjects. Solid lines: right eye dominance at center. Dashed lines: left eye dominance at center. (B) Same data shifted so that the $50 \%$ crossover point aligns with zero on the abscissa. The thick line shows the average across all subjects. Modified from Khan and Crawford, 2001a.

would map the retinal vector directly onto a motor vector. One obvious flaw with coding arm motor commands this way is that most of the time the arm does not start aligned with gaze. When we go from a resting position to reach for a target, we are generally accurate, even when we do not have visual feedback of the arm and target (e.g. Crawford et al., 2000; Henriques and Crawford, 2000). The only way to do this is by combining the visual signal with an internal sense of eye position (von Helmholtz, 1867; Mittelstaedt, 1983).

The recognition that the brain needs to take eye position into account when computing target direction has led some theories to suggest that the brain might approximate the required arm/eye rotation by a simple vector addition - merely adding all reti- nal vectors onto a vector coding initial eye direction (Hallett and Lightstone, 1976; Mays and Sparks, 1980; Flanders et al., 1992). But this vector-addition strategy would lead to marked errors in some situations because displacements in retinal coordinates are not the same as displacements in head or shoulder coordinates (Crawford and Guitton, 1997). For example, the way that straight lines project onto the retina depends on eye position in a complex manner. In Fig. 5, we have two earth-fixed, horizontal arcs centered on an eyeball. In Fig. 5A, the eye is fixating a diamond at eye-level, while in Fig. 5B, the eye is fixating a diamond $30^{\circ}$ up. For each diamond, a square is placed $60^{\circ}$ to its right, so that both objects lie on the same horizontal arc in space. The left and right columns show how the images of these objects project on the retinal for two different perspectives. When the eye fixates the eye-level diamond (Fig. 5A), the retinal image of the eye-level square falls on the retinal meridian (dashed line on the back of the transparent eye). But when the eye fixates the diamond $30^{\circ}$ up (Fig. 5B), the retinal image of the square falls below the horizontal meridian of the eye - owing to the upward rotation of the eye. Thus, spatial displacements that might be visually horizontal (i.e. on the retina) at one eye position become oblique at other eye positions, whereas in head/shoulder coordinates they stay the same. If the brain tried to compute these object's location relative to the head or the shoulder simply by using vector addition, it would misestimate the elevations of at least some objects, and would misaim resultant eye and arm movements.

To test the way that the eye-hand coordination system handles this problem, we had subjects point between various horizontally displaced targets, in the absence of visual feedback (Crawford et al., 2000). What we found was that: (1) the projections of these target displacements onto the retina varied strongly with eye position in a complex, non-linear fashion; and (2), despite this, pointing movements were essentially accurate. In other words, the visuomotor transformation for pointing did not just take the 'first-order' aspects of eye position into account (i.e. adding the visual vector to an eye position vector), it in effect accounted for 3-D eye rotation and performed the correct non-linear reference frame transformation. 

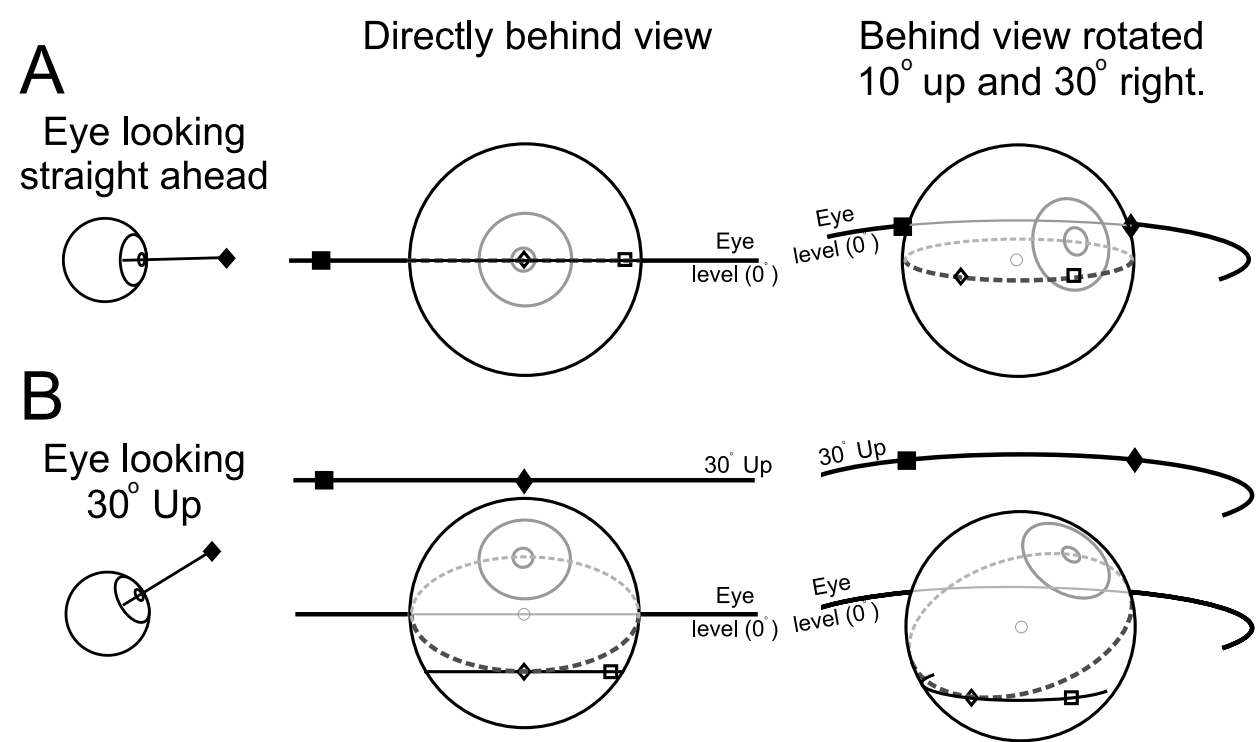

Fig. 5. Simulated eye position-dependent geometry of retinal stimulation. Two views of the eyeballs and targets are shown: one directly behind edge-on (left column) and one behind and slightly up and to the right (right column). The eyeball is transparent, so we can see its nodal point (small circle), its horizontal meridian (dashed sphere), the two external objects (closed symbols) and their retinal projections on the back of the eye (open symbols). For both eye positions (A, eye directed straight ahead; and B, eye rotated $30^{\circ}$ upward), the eye is fixating a diamond target. A square is located $60^{\circ}$ to the right of the diamond at the same elevation, on a horizontal arc (wrapped around the eye) at: eye level (A) and $30^{\circ}$ up (B). When the eye looks at the eye-level diamond (A), the retinal image of the square lies on the horizontal meridian. But when the eye looks up at the diamond on the top arc (B), the image of the square no longer falls on the horizontal meridian.

\section{Using your head}

Similar arguments hold for taking head orientation into account. For example, if one were to purposefully keep the eyes centered while looking around, the head moves pretty much like an eye (Ceylan et al., 2000) and at least for far targets, the geometry described in the previous section holds pretty much the same: one needs to take head orientation into account in a non-linear fashion.

But head movement has other, special implications for eye-hand coordination. This is because the centers of rotation of the eye, head and shoulder do not coincide. As a result, each time the head rotates, it causes the eyes to translate through space relative to the shoulder, which changes the angular direction of targets relative to the eye (but not the shoulder). One could rely on visual feedback of the hand during pointing or reaching - to make up for any differences this might make, but we were interested to see if the system took this into account without such feedback.
To do this, we had subjects point toward distant targets with the head in different horizontal positions (Henriques and Crawford, 2002). This caused significant changes in the line from the eye to the target (recall that this is the line that subjects use for aligning pointing when looking straight ahead). Nevertheless, subjects were able to re-calculate this line, and accurately place the fingertip at the right location for any head position. In a related experiment (Henriques et al., 2001) we found that subjects were similarly able to account for head orientation and the resulting translation of eye location when computing the visual angle of near targets for reaching movements. These results show not only that head orientation is taken into account, but also, moreover, that the eye-hand coordination system possesses a sophisticated representation of body geometry that takes into account the differences in the centers of rotation of the eyes, head, and shoulder in the absence of on-line visual feedback. Such a representation is likely constructed or at least fine-tuned by learning. 


\section{Arms and the man}

Eye-hand coordination must end in a movement of the hand. But at what point in the brain is the transition from mechanisms specific to eye-hand coordination to those purely associated with arm control? A reasonable dividing line would be the point where gaze and head signals no longer influence neural firing rates, where these are purely modulated by the kinematics and dynamics of the arm movement. Most of the current evidence suggests that this has not yet occurred at the level of premotor cortex (Mushiake et al., 1997), but is completed by or within M1 (Mushiake et al., 1997; Crammond and Kalaska, 2000).

By corollary, one might not expect eye-hand coordination to have much to do with the details of arm kinematics that are not related to aiming the hand or conforming grasp to the target. A prime example would be the neural constraints to determine redundant degrees of freedom in the arm control system (Bernstein, 1967). Nevertheless, (without going into detail) there are considerable similarities between some of the three-dimensional kinematics constraints observed in the upper arm and in the eye (Straumann et al., 1991; Theeuwen et al., 1993). Based on this, it was once suggested that these constraints were developed in unison in order to facilitate coordination of the eye and arm in the workspace (Straumann et al., 1991).

In order to test this, we dissociated gaze from arm movements and measured the three-dimensional constraints in the latter (Medendorp et al., 2000). In a similar situation, we had previously found that dissociating gaze from head movement disrupted the normal 3-D control of the head, demonstrating the intimate coupling of these two systems (Ceylan et al., 2000). However, the same did not occur: although dissociating gaze from the arm again led to a disruption in movement accuracy, it had no effect on the three-dimensional constraints on arm orientation (Medendorp et al., 2000). Based on these findings, we conclude that such constraints are organized in neural structures downstream from those which employ gaze signals to compute arm movement direction.

\section{Synthesis and conclusion}

As stated in the introduction, the first aim of this work is to create a model of eye-hand coordination that can help us to understand visually directed arm movements, and help guide our neurophysiological investigations. The working model that we have been using for the past few years is illustrated in Fig. 6 . Admittedly it is cartoonish in several respects: in its reliance on forward serial transformations (we all know the brain is really heavily parallel and recurrent in organization), in its representation of signals (no one should expect such discrete, explicit representations in the brain), and in its simplicity (even many aspects of the work described here have been left out). And yet in outline, it agrees with much of the known physiology.

This model might also account for some symptoms observed in the neuropsychology literature. For example, Carey et al. (2000) report a patient who reaches 'magnetically' toward the direction of fixation. Our model would predict this if the extra-foveal portions of the early gaze-centered representation for arm movement (i.e. perhaps the parietal reach region) were lost (a condition not unlikely given the tendency to over-represent extra-foveal space). If only activity in the foveal zone were possible, the downstream transformations would automatically convert this into a reaching movement toward the fixation object (and conversely would be unable to reach toward non-foveal objects).

A key feature of this model is its use of early representations of attended target locations in a retinal frame, representations that must be updated with each eye movement. Only the target that is selected for action is put through the subsequent transformations: comparisons with eye and head orientations, computation of inverse kinematics and dynamics of the arm and so on. From a biological perspective, this is a space and energy saving feature: there would be an enormous cost to try to perform these operations on every point in space, and very little advantage. This goes a long way toward explaining why the early stages of arm control seem to be organized in eye-centered coordinates (Henriques et al., 1998). But it also has a surprising implication. Whereas introspection may suggest that we have a complete map of visuomotor space, this model suggests that 


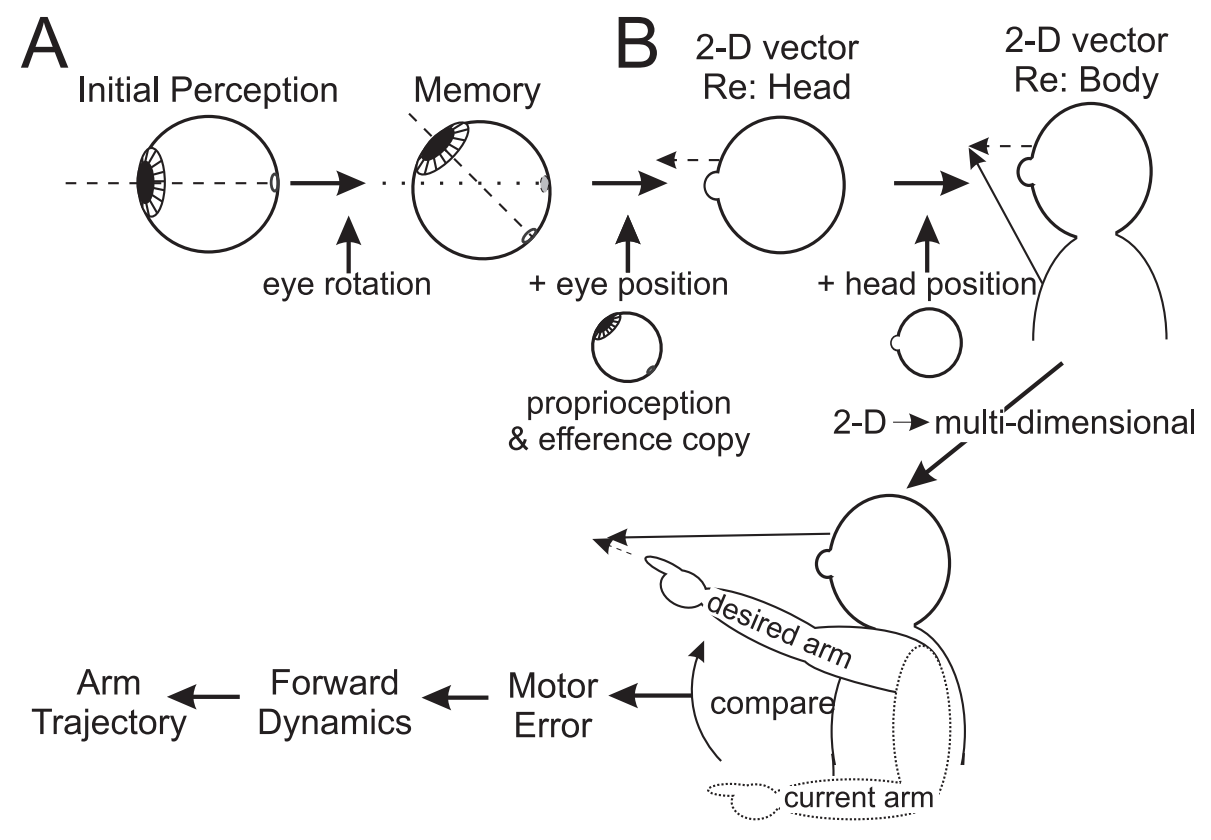

Fig. 6. Conversion-on-demand hypothesis of visuomotor representation and visuomotor control. Target representations are initially held in an eye-centered frame, and can be shifted in this frame to compensate for intervening eye movements through dynamic remapping (A). Once a target has been selected for action, its final retinal representation is put through a reference frame transformation (by comparing it with 3-D orientation of eyes and head) to generate an accurate 3-D motor signal in motor reference frame (B). Modified from Henriques et al., 1998.

this is an illusion - only the overall transformation provides such a map, and only on those representations we choose to act on. The catch is that every time we test our intuition by choosing to act on a representation, it confirms our intuition of a complete map. In this sense, this is a 'virtual' map of space, similar to ideas that have been proposed for visual perception (Howard, 1982).

A less realistic aspect of the cartoon in Fig. 6 is the suggestion of explicit signals of desired target location relative to the head, etc. These are clearly not found in actual physiology, which tends to employ retinal codes (Gnadt and Andersen, 1988; Sparks, 1989; Duhamel et al., 1992). However, when one simulates the same transformations by training a neural net to transform a visual vector into a motor vector (Smith and Crawford, 2001a,b), one finds that the network learns to do so through fairly simple position modulations, resulting in an organization that captures the basic conceptual flow of Fig. 6, while using signals that resemble actual physiol- ogy. Thus, as originally suggested by Andersen and colleagues, reference frame transformations can be explicit (Zipser and Andersen, 1988), but in fact one can entirely get rid of the need for position maps for a more realistic model. Indeed, models that do not rely on explicit position codes turn out to be more flexible and powerful (Ceylan et al., 2000). Incorporating these ideas, and all of the findings described above in this article into a complete computational model following the outline provided in Fig. 6 will be a major goal of our future work.

\section{Acknowledgements}

This research is supported by CIHR group grant, Canadian NSERC and PREA. J.D.C is supported by the Canadian Research Chair program, D.Y.P.H is supported by an E.A Baker Foundation CIHR Research Doctorate Award, and W.P.M. is supported by the $\mathrm{Hu}-$ man Frontiers Science Program. 


\section{References}

Andersen, R.A. and Mountcastle, V.B. (1983) The influence of the angle of gaze upon the excitability of the light-sensitive neurons of the posterior parietal cortex. J. Neurosci., 3: 532548.

Batista, A.P., Buneo, C.A., Snyder, L.H. and Andersen, R.A. (1999) Reach plans in eye-centered coordinates. Science, 285: 257-260.

Bernstein, N. (1967) The Coordination and Regulation of Movements. Pergamon Press, Oxford.

Bock, O. (1986) Contribution of retinal versus extraretinal signals towards visual localization in goal-directed movements. Exp. Brain Res., 64: 467-482.

Bremmer, F., Graf, W., Ben-Hamed, S. and Duhamel, J.R. (1999) Eye position encoding in the macaque ventral intraparietal area (VIP). NeuroReport, 10: 873-878.

Carey, D.P., Della Sala, S. and Ietswart, M. (2002) Neuropsychological perspectives on eye-hand coordination in visually guided reaching. In: J. Hyönä, D.P. Munoz, W. Heide and R. Radach (Eds.), The Brain's Eye: Neurobiological and Clinical Aspects of Oculomotor Research. Progress in Brain Research, Vol. 140. Elsevier, Amsterdam, pp. 000-000.

Ceylan, M.Z., Henriques, D.Y.P., Tweed, D.B. and Crawford, J.D. (2000) Task-dependent constraints in motor control: pinhole goggles make the head move like an eye. J. Neurosci., 20: 2719-2730.

Colby, C.L. and Goldberg, M.E. (1999) Space and attention in parietal cortex. Annu. Rev. Neurosci., 22: 319-349.

Coren, S. and Kaplan, C.P. (1973) Patterns of ocular dominance. Am. J. Optom. Arch. Am. Acad. Optom., 50: 283-292.

Crammond, D.J. and Kalaska, J.F. (2000) Prior information in motor and premotor cortex: activity during the delay period and effect on pre-movement activity. J. Neurophysiol., 84: 986-1005.

Crawford, J.D., Henriques, D.Y.P. and Vilis, T. (2000) Curvature of visual space under vertical eye rotation: implications for spatial vision and visuomotor control. J. Neurosci., 20: 23602368.

Crider, B.A. (1944) A battery of tests for the dominant eye. $J$. Gen. Psychol., 31: 179-190.

Duhamel, J.-R., Colby, C.L. and Goldberg, M.E. (1992) The updating of the representation of visual space in parietal cortex by intended eye movements. Science, 255: 90-92.

Engel, K.C., Anderson, J.H. and Soechting, J.F. (2000) Similarity in the response of smooth pursuit and manual tracking to a change in the direction of target motion. J. Neurophysiol., 84: 1149-1156.

Enright, J.P. (1995) The non-visual impact of eye orientation on eye-hand coordination. Vision Res., 35: 1611-1618.

Fisk, J.D. and Goodale, M.A. (1985) The organization of eye and limb movements during unrestricted reaching to targets in contralateral and ipsilateral visual space. Exp. Brain Res., 60: 159-178.

Flanders, M., Helms-Tillery, S.I. and Soechting, J.F. (1992) Early stages in a sensorimotor transformation. Behav. Brain Sci., 15: 309-362.
Fu, Q.F., Suarez, J.I. and Ebner, T.J. (1993) Neuronal specification of direction and distance during reaching movements in the superior precentral premotor area and primary motor cortex of monkeys. J. Neurophysiol., 70: 2097-2116.

Gielen, C.C., van den Heuvel, P.J. and van Gisbergen, J.A. (1984) Coordination of fast eye and arm movements in a tracking task. Exp. Brain Res., 56: 154-161.

Gnadt, J.W. and Andersen, R.A. (1988) Memory related motor planning activity in posterior parietal cortex of macaque. Exp. Brain Res., 70: 216-220.

Graziano, M.S., Yap, G.S. and Gross, C.G. (1994) Coding of visual space by premotor neurons. Science, 152: 1603-1608.

Hallett, P.E. and Lightstone, A.D. (1976) Saccadic eye movements to flashed targets. Vision Res., 16: 107-114.

Henriques, D.Y.P. and Crawford, J.D. (2000) Direction dependent distortions of retinocentric space in the visuomotor transformation for pointing. Exp. Brain Res., 132(2): 179-194.

Henriques, D.Y.P. and Crawford, J.D. (2002) Role of eye, head and shoulder geometry in the planning of accurate arm movements. J. Neurophys., 87: 1677-1685.

Henriques, D.Y.P., Klier, E.M., Smith, M.A., Lowey, D. and Crawford, J.D. (1998) Gaze-centered re-mapping of remembered visual space in an open-loop pointing task. J. Neurosci., 18: 1583-1594.

Henriques, D.Y.P., Crawford, J.D., Medendorp, W.P. and Gielen, C.C.A.M. (2001) The eye-hand coordination system accounts for head orientation and target depth during reaching toward near targets. Abstr. Soc. Neurosci., 27(2).

Howard, I.P. (1982) Human Visual Orientation. Wiley, New York.

Jouffrais, C. and Boussaoud, D. (1999) Neuronal activity related to eye-hand coordination in the primate premotor cortex. Exp. Brain Res., 128: 205-209.

Khan, A.Z. and Crawford, J.D. (2001a) Ocular dominance reverses as a function of gaze angle. Vision Res., 41: 17431748.

Khan, A.Z. and Crawford, J.D. (2001b) Coordinating one hand with two eyes: gaze-dependent reversal of ocular dominance in a pointing task. Abstr. Soc. Neurosci., 940.12.

Mays, L.E. and Sparks, D.L. (1980) Saccades are spatially, not retinotopically coded. Science, 208: 1163-1164.

Mazzoni, P., Bracewell, R.M., Barash, S. and Andersen, R.A. (1996) Spatially tuned auditory responses in area LIP of macaques performing delayed memory saccades to acoustic targets. J. Neurophysiol., 75: 1233-1241.

Medendorp, W.P. and Crawford, J.D. (2002) Visuospatial updating of reaching target in near and far space. NeuroReport, 13: 633-636.

Medendorp, W.P., Crawford, J.D., Henriques, D.Y.P., Van Gisbergen, J.A.M. and Gielen, C.C.A.M. (2000) Kinematic strategies for upper arm - forearm coordination in three dimensions. $J$. Neurophysiol., 84: 2302-2316.

Miles, W.R. (1930) Ocular dominance in human adults. J. Gen. Psychol., 3: 412-420.

Mittelstaedt, H. (1983) A new solution to the problem of the subjective vertical. Naturwissenschaften, 70: 272-281.

Mushiake, H., Tanatsugu, Y. and Tanji, J. (1997) Neuronal activ- 
ity in the ventral part of premotor cortex during target-reach movement is modulated by direction of gaze. J. Neurophysiol., 78: 567-571.

Ono, H. and Barbeito, R. (1982) The cyclopean eye vs. the sighting-dominant eye as the center of visual direction. Percept. Psychophys., 32(3): 201-210.

Porac, C. and Coren, S. (1976) The dominant eye. Psychol. Bull., 83(5): 880-897.

Riehle, A. and Requin, J. (1995) Neuronal correlates of the specification of movement direction and force in four cortical areas of the monkey. Brain Behav. Res., 70: 1-13.

Schlag, J., Schlag-Rey, M. and Pigarev, I. (1992) Supplementary eye field: influence of eye position on neural signals of fixation. Exp. Brain Res., 90: 302-306.

Smith, M.A. and Crawford, J.D. (2001a) Network properties in a physiologically realistic model of the 2-D to 3-D visuomotor transformation for saccades. Abstr. Soc. Neurosci., 27(1).

Smith, M.A. and Crawford, J.D. (2001b) Self-organizing task modules in explicit coordinate systems in a neural network model for 3-D saccades. J. Comput. Neurosci., 10: 127-150.

Sparks, D.L. (1989) The neural encoding of the location of targets for saccadic eye movements. J. Exp. Biol., 146: 195-207.
Straumann, D., Haslwanter, T., Hepp-Reymond, M.C. and Hepp, K. (1991) Listing's law for eye, head and arm movements and their synergistic control. Exp. Brain Res., 86: 209-215.

Theeuwen, M., Miller, L.E. and Gielen, C.C.A.M. (1993) Is the orientation of head and arm coupled during pointing movements?. J. Motor Behav., 25: 242-250.

Vercher, J.-L., Magenes, G., Prablanc, C. and Gauthier, G.M. (1994) Eye-head-hand coordination in pointing at visual targets: spatial and temporal analysis. Exp. Brain Res., 99: 507523.

von Helmholtz, H. (1867) Handbuch der Physiologischen Optik, Bd. 3. Voss, Hamburg, Germany.

von Holst, E. and Mittelstaedt, H. (1950) Das Reafferenzprinzip (Wechselwirkungen zwischen Zentralnervensystem und Peripherie). Naturwissenschaften, 37: 464-476.

Wells, W.C. (1792) An Essay Upon Single Vision with Two Eyes: Together with Experiments and Observations on Several Other Subjects in Optics. Cadell, London.

Zipser, D. and Andersen, R.A. (1988) A back-propagation programmed network that simulates response properties of a subset of posterior parietal neurons. Nature, 331: 679-684.

\section{QUERIES:}

?\#1: Crawford and Guitton, 1997 not in reference list (page 335) ?\#2: Carey et al. (2000) not in reference list (page 337)

?\#3: Sparks, '1991' $\rightarrow$ '1989'. If incorrect, please supply ref. for 1991 and cite 1989 (page 338)

?\#4: von Holst and Mittelstaedt, 1950: not referred to in text. (page 338) 